\title{
Derivation of postharvest fruit behavior reduced order models for on-line monitoring and control of quality parameters during refrigeration
}

December 3, 2012

\author{
Diana Rivas ${ }^{1}$, Carlos Vilas ${ }^{* 1}$, Antonio A. Alonso ${ }^{1}$, Fernando Varas ${ }^{2,3}$ \\ ${ }^{1}$ Process Engineering Group, IIM-CSIC, Eduardo Cabello, 6, 36208 Vigo, Spain \\ 2 Applied Mathematics Department, School of Telecommunication Engineering, \\ Universidad de Vigo, Campus Marcosende, 36310 Vigo, Spain \\ ${ }^{3}$ Universidad Politécnica de Madrid, Plaza Cardenal Cisneros, 28040 Madrid, Spain \\ * Corresponding author: carlosvf@iim.csic.es
}

\begin{abstract}
In this paper an efficient computational tool to predict the evolution of the key variables in the fruit refrigeration process is presented. This computational model is intended to be used in real time quality control.

The tool is based on detailed mathematical models proposed in the literature which accounts for the main phenomena occurring during refrigeration. Since integration using standard numerical schemes leads to a computational cost unaffordable in real time environments, a reduced order model (able to combine a high accuracy with a low computational cost) is derived.

In the derivation of this model, the role of the presence of quite disparate time scales is considered. In this respect, two alternative reduced order models are considered: a general model (aiming at describing the evolution at arbitrary time scales) and a model assuming
\end{abstract}


a strong coupling between the state variables (as expected in the long

term behavior of the system).

Keywords: fruit storage, refrigeration process, fruit quality, on-line monitoring and control, reduced order models

\section{Practical Applications}

The modeling reduction methodology proposed in this work for the fruit refrigeration process, either during storage or transport, will enable the use of CAPE tools for reducing the computational load required to perform predictions. With such tools, optimal operation policies to maximize fruit quality, extend the storage time and/or minimize costs can be efficiently obtained. Also, in the event of unexpected disturbances, they will allow us to recompute in real time the operation policy, and in this way ensure a minimum quality lost.

\section{Introduction}

Refrigeration is probably the most critical process in fruit storage and transport. A poor temperature/pressure control inside the storage chamber or the cargo would imply a quality loss of the fruit. In general, industrial operation policies in this kind of processes are still guided by highly empiric recipes and rules of thumb which are usually too rigid to adapt the operation to sudden changes in the production conditions or process disturbances. There exists, therefore, a real need for decision making tools based on Computed Aided Process Engineering (CAPE) to help us to design the operation policies. Mathematical models are usually key components of CAPE which, in the context of online monitoring and control, need to be run in real time environments and therefore be as efficient as possible.

In recent years, detailed first principles mathematical models describing the different phenomena involved in the refrigeration process have been developed and validated using different experimental techniques to measure the key state variables -see De Smedt et al. (2002); Nguyen et al. (2006); Tri Ho et al. (2006, 2008)-. Water permeation through the tissues, heat and mass transport or biochemical transformations such as those related to respiration, are some of the fruit refrigeration phenomena considered in the literature. 
One important feature of this kind of processes is the relevance of the spatial distribution of several state variables (like temperature or gas concentration) involved in the different mechanisms of the process. Furthermore the complex chemical reactions taking place make the system highly nonlinear. As a result, mathematical descriptions of these processes consist of sets of nonlinear partial differential equations (PDEs). Classical numerical techniques for solving PDEs, based on spatial discretization schemes (like finite element methods, finite difference methods or finite volumen methods), were employed in the literature to simulate the fruit refrigeration process (Nguyen et al., 2006; Tri Ho et al., 2006, 2008). Such classical techniques employ the values of the variables at each mesh node as the degrees of freedom in the discrete numerical model. As a consequence, the resulting set of ordinary differential equations (ODEs) is computationally involved, especially in 2D or 3D spatial domains with realistic geometries, due to its high dimensionality and the complexity of the nonlinear terms. Therefore, direct use of these techniques could result unsuitable for real time monitoring and control.

Reduced order models (ROM) emerged as an efficient alternative to the classical techniques for real time applications. These techniques are based on projection of the original PDEs system over a set of globally defined basis functions (Sirovich, 1987; Balsa-Canto et al., 2002; Alonso et al., 2010). Depending on the way of computing the basis functions, different techniques arise, for instance the proper orthogonal decomposition (POD) or laplacian spectral decomposition (for more details see Vilas et al. (2006)) to name a few. We choose, in this work, the POD method because it is optimal in the sense that, for a given number of basis functions, it captures most of the relevant dynamic behavior of the original distributed system in the range of initial conditions, parameters, inputs and/or perturbations of the experimental data (Holmes et al., 1997).

When constructing the ROM with the POD technique, a set of snapshots (numerical values of the spatio-temporal evolution of the representative state variables) must be collected to compute the set of basis functions for each of the state variables (Park and Chung, 2000; Gunes, 2002; Padhi and Balakrishnan, 2003; Vilas et al., 2008). In the sequel, we will refer to this approach as separate basis. However one could take advantage of the coupling effects due mainly to the reaction terms, which make the evolution of some of the state variables strongly connected to the behavior of the others. This property will be employed in the second approach, joint basis, to derive a unique set of basis functions and, in this manner, to reduce even more the dimen- 
sionality of the original model. In both approaches the set of snapshots must be collected in the range of the process operating conditions.

In this contribution, the proposed reduction schemes will be validated by simulation experiments on a model system representative of fruit behavior during refrigeration. The kinetic expressions, transport and reaction, have been taken from literature. The model will be used to obtain the collection of snapshots required to compute the PODs. It must be noted, however, that the proposed technique is expected to work as well with experimental data, obtained for instance from magnetic nuclear resonance (Nguyen et al., 2006).

One of the main difficulties in the derivation of ROMs lies in computing the integrals of the nonlinear terms which appear as a result of the projection procedure. Some options include: Simpson's rule; the fast Fourier transform (Gottlieb and Orszag, 1977) in combination with a collocation method (Theodoropoulou et al., 1998) or neural networks (Rico-Martínez et al., 1995). The main inconvenience of the Simpson's rule is that it usually turns out to be computationally expensive. On the other hand, the fast Fourier transform could result into a large number of ODEs, particularly in $2 \mathrm{D}$ or $3 \mathrm{D}$ spatial geometries. Finally, the problem with neural networks lies in the laborious work required to train the network. We propose, in this paper, an alternative and efficient technique based on the finite element structure.

This work is structured as follows: in section 2, the complete mathematical model, including the different phenomena involved in the process, is described. Model reduction techniques based on both separate and joint basis of the proper orthogonal decomposition will be presented in Section 3. Main results of the work including formal validation of the ROM will be discussed in Section 4 on a number of simulation experiments. Finally, the main conclusions together with future lines of research will be summarized in Section 5.

\section{Materials and Methods}

\subsection{Mathematical model}

The aim of this section is to present a detailed mathematical model describing the main phenomena occurring during refrigeration of fruit (in particular pears). Such model considers the following processes: hydrolysis of starch 
and middle lamella; cellular respiration; fermentation; as well as gas, water and heat transport. The chemical reactions that take place are:

$$
\begin{gathered}
\left(\mathrm{C}_{6} \mathrm{H}_{10} \mathrm{O}_{5}\right)_{n_{S}}+n_{S} \mathrm{H}_{2} \mathrm{O} \stackrel{r_{S}}{\longrightarrow} n_{S} \mathrm{C}_{6} \mathrm{H}_{12} \mathrm{O}_{6} \\
\mathrm{C}_{6} \mathrm{H}_{12} \mathrm{O}_{6}+6 \mathrm{O}_{2} \stackrel{r_{H}}{\longrightarrow} 6 \mathrm{CO}_{2}+6 \mathrm{H}_{2} \mathrm{O} \\
\mathrm{C}_{6} \mathrm{H}_{12} \mathrm{O}_{6} \stackrel{r_{F}}{\longrightarrow} 2 \mathrm{C}_{2} \mathrm{H}_{5} \mathrm{OH}+2 \mathrm{CO}_{2} \\
\text { Lamella }+\mathrm{H}_{2} \mathrm{O} \stackrel{r_{L}}{\longrightarrow} \text { Pectine }
\end{gathered}
$$

In our case, the model comprises eight state variables: temperature $(T)$, concentration of starch $\left(C_{S}\right)$, middle lamella $\left(C_{L}\right)$, hexose $\left(C_{H}\right)$ (considered as the only product of starch hydrolysis), water $\left(C_{W}\right)$, oxygen $\left(C_{\mathrm{O}_{2}}\right)$, carbon dioxide $\left(C_{\mathrm{CO}_{2}}\right)$ and nitrogen $\left(C_{N_{2}}\right)$. The model has been built based on the bibliographic sources available which include experimental model validation. In this regard, biochemical reaction rates as well as water transfer process have been taken from De Smedt et al. (2002) and Lammertyn et al. (2003). The works by Tri Ho et al. $(2006,2008)$ have been employed to describe the gas transport in cellular tissue using a permeation-diffusion-reaction model while the model for water transport in pear has been obtained from Nguyen et al. (2006). The heat transfer is described by the Fourier equation whose parameters have been taken from Martínez-Monzo et al. (1999). The complete set of equations for the fruit refrigeration process reads as follows:

$$
\begin{aligned}
\frac{\partial C_{S}}{\partial t} & =r_{S} ; \quad C_{S}\left(t_{0}\right)=C_{S_{0}} \\
\frac{\partial C_{H}}{\partial t} & =-n_{S} r_{S}+\frac{r_{H}}{6} ; \quad C_{H}\left(t_{0}\right)=C_{H_{0}} \\
\frac{\partial C_{L}}{\partial t} & =r_{L} ; \quad C_{L}\left(t_{0}\right)=C_{L_{0}} \\
\alpha_{C_{O_{2}}} \frac{\partial C_{O_{2}}}{\partial t}+\nabla\left(\overline{\mathbf{u}} C_{O_{2}}\right) & =\nabla\left(D_{C_{O_{2}}} \nabla C_{O_{2}}\right)+r_{H} ; \quad C_{O_{2}}\left(t_{0}\right)=C_{O_{2,0}} \\
\alpha_{C_{C O_{2}}} \frac{\partial C_{C O_{2}}}{\partial t}+\nabla\left(\overline{\mathbf{u}} C_{C O_{2}}\right) & =\nabla\left(D_{C_{C O_{2}}} \nabla C_{C O_{2}}\right)-R Q r_{H}+r_{F} ; \quad C_{C O_{2}}\left(t_{0}\right)=C_{C}(5,2) \\
\alpha_{C_{N_{2}}} \frac{\partial C_{N_{2}}}{\partial t}+\nabla\left(\overline{\mathbf{u}} C_{N_{2}}\right) & =\nabla\left(D_{C_{N_{2}}} \nabla C_{N_{2}}\right) ; \quad C_{N_{2}}\left(t_{0}\right)=C_{N_{2,0}} \\
\frac{\partial C_{W}}{\partial t} & =\nabla\left(D_{W} \nabla C_{W}\right)+r_{S}+r_{L}-r_{H} ; \quad C_{W}\left(t_{0}\right)=C_{W_{0}} \\
\rho c_{T} \frac{\partial T}{\partial t} & =\nabla\left(K_{T} \nabla T\right) ; \quad T\left(t_{0}\right)=T_{0}
\end{aligned}
$$


where $\nabla$ denotes the standard gradient operator and $\alpha_{C_{i}}$ represents the gas capacity (Tri Ho et al., 2006). Those equations involving transport are completed with the following boundary conditions:

$$
\begin{aligned}
D_{C_{i}} \frac{\partial C_{i}}{\partial n} & =h_{C_{i}}\left(C_{i}^{\infty}-C_{i}\right), \quad i \in\left\{W, O_{2}, C O_{2}, N_{2}\right\} \\
K_{T} \frac{\partial T}{\partial n} & =h_{T}\left(T^{\infty}-T\right)
\end{aligned}
$$

with $D_{C_{i}}$ being the diffusion coefficients, $h_{C_{i}}$ the mass transfer coefficient, $\rho$ the density, $c_{T}$ the specific heat capacity, $K_{T}$ the thermal conductivity and $h_{T}$ the transfer coefficient for temperature. Nonlinear terms related to chemical reactions are of the form:

$$
\begin{gathered}
r_{S}=-k_{S} C_{S} \frac{C_{W}}{C_{S}+C_{H}+C_{W}} \\
r_{L}=-k_{L} C_{L} \frac{C_{W}}{C_{L}^{0}+C_{P}^{0}+C_{W}} \\
r_{H}=-V_{m, O_{2}, r e f} \frac{\exp \left[\frac{E_{a, V m, O_{2}}}{R}\left(\frac{1}{T_{r e f}}-\frac{1}{T}\right)\right] C_{O_{2}}}{\left(K_{m, O_{2}}+C_{O_{2}}\right)\left(1+\frac{C_{C O_{2}}}{K_{m n, C O_{2}}}\right)} \\
r_{F}=V_{C O_{2, r e f}} \frac{\exp \left[\frac{E_{a, V m f C O_{2}}}{R}\left(\frac{1}{T_{r e f}}-\frac{1}{T}\right)\right]}{\left(1+\frac{C_{O_{2}}}{K_{m, f, O_{2}}}\right)}
\end{gathered}
$$

where $C_{L}^{0}$ and $C_{P}^{0}$ represent, respectively, the initial concentrations of middle lamella and pectine.

Finally the permeation velocity vector, $\overline{\mathbf{u}}$, is described by Darcy's law (Tri Ho et al., 2006; Datta, 2007):

$$
\overline{\mathbf{u}}=-\frac{k_{p}}{\mu} \nabla P=-\frac{k_{p} R T}{\mu} \nabla\left(\sum C_{i}\right),
$$

where $k_{p}$ is the permeation coefficient, $P$ the pressure, $\mu$ the gas viscosity and index $i=\left\{\mathrm{O}_{2}, \mathrm{CO}_{2}, \mathrm{~N}_{2}\right\}$. The description and value of the parameters and constants employed in these equations are presented in Tables 1 and 2. 


\subsection{Reduced order models: The proper orthogonal de- composition}

\subsubsection{Overview}

In order to solve a system of PDEs as the one presented in the previous section, numerical methods are required. Classical numerical techniques like the finite element or the finite differences methods (see, for instance, Schiesser (1991); Reddy (1993)) usually result into a large number of ODEs which make these approaches unsuitable for applications such as optimization or real-time control. As an alternative to classical methods, we propose here the use of ROMs and, in particular, the POD technique which is briefly described in the sequel.

Usually when considering a system with several state variables, a different set of POD basis is computed for each of them. In this work we will call this approach separate basis. However, under certain circumstances it may result more convenient to compute a unique set of POD basis containing information about all the system variables (joint basis). Next, both approaches are presented.

\subsubsection{Separate basis}

Like many other numerical techniques for PDE systems, the ROM approach proposed here approximates, in a first step, each system variable by a truncated series of the form:

$$
z_{i}(\xi, t) \simeq \tilde{z}_{i}(\xi, t)=\sum_{j=1}^{p_{i}} m_{i, j}(t) \phi_{i, j}(\xi),
$$

where $\left\{\phi_{i, j}(\xi)\right\}_{j=1}^{p_{i}}$ is the basis set and $\left\{m_{i, j}(t)\right\}_{j=1}^{p_{i}}$ are the coefficients of the series decomposition. The subindex $i$ refers to each of the system states $\left\{C_{S}, C_{H}, C_{L}, C_{W}, C_{O_{2}}, C_{C O_{2}}, C_{N_{2}}, T\right\}$. One of the key differences between this approach and classical numerical methods like the FEM is that the basis functions $\phi_{i, j}(\xi)$ are globally defined in the spatial domain. It is worth noting that, in the POD technique, the larger the number of elements $p_{i}$ in Eqn (16), the better the approximation $z_{i}(\xi, t) \simeq \tilde{z}_{i}(\xi, t)$ (García et al., 2012). In fact when $p_{i} \rightarrow \infty$ the original field is recovered (Fletcher, 1984; Vilas et al., 2006). 
In order to obtain the approximated solution $\tilde{z}_{i}(\xi, t)$ both the basis functions $\left(\phi_{i, j}(\xi)\right)$ and the time dependent coefficients $\left(m_{i, j}(t)\right)$ must be known beforehand. Let us first start with the basis functions which, in the POD case, are computed as the solution of the following eigenvalue problem:

$$
\lambda_{i, j} \phi_{i, j}(\xi)=\int_{V} K_{i}\left(\xi, \xi^{\prime}\right) \phi_{i, j}\left(\xi^{\prime}\right) d \xi^{\prime}
$$

where $\lambda_{i, j}$ corresponds with the eigenvalue associated with each global basis function $\phi_{i, j}$. An important property of the eigenvalues obtained from (17) is that they can be ordered so that $\lambda_{i, j} \geq \lambda_{i, l}$ for $j<l$ and $\lambda_{i, \ell} \rightarrow 0$ as $\ell \rightarrow \infty$.

For practical reasons, instead of working with the continuous version of the kernel $K_{i}\left(\xi, \xi^{\prime}\right)$, we use its discrete version, i.e., a symmetric and positive definite matrix of the form:

$$
K_{i}=\frac{1}{k} \sum_{n=1}^{k} Z_{i, n} Z_{i, n}^{T}
$$

with $Z_{i, n} \in \mathbf{R}^{N}$ representing the vector of measurements of state $i$ at a finite number $N$ of spatial points and at a given time $t_{n}$ (snapshot). The summation in (18) extends over a sufficiently rich collection of uncorrelated snapshots $n=1, . ., k$. These can be obtained either from experiments or by direct numerical simulation of the original PDE. Note that, in this approach, a POD basis set is computed for each state variable.

Choosing the vector of state measurements is one of the most important parts in the construction of a reduced order model with the POD technique. This selection will have a direct impact on the capability of the ROM to reproduce the system behavior as well as on the dimensionality of the ROM. In this regard, it is important to mention that the whole range of the process operating conditions should be covered when taking the snapshots. Also, if the objective is to capture fast changes in the dynamics, most measurements should be taken when such changes occur. On the contrary, when the possible steady states are the most representative parts of the process, filling the set of snapshots with a large number of measurements taken at fast changing dynamic regions could result unproductive. The snapshots in this work will be generated by simulation experiments through the finite element method.

Regarding the resolution of Eqn (17), it must be pointed out that, for a large number $N$ of spatial measurements, it may result computationally involved. In order to avoid this problem, a useful alternative proposed by 
Sirovich (1987) and known as the method of snapshots or strobes can be employed. It should be noted that the computation of the POD basis is performed off-line, therefore the time required to carry out this task has no influence when implementing the procedure on a real time scheme.

Once the basis functions in Eqn. (16) have been computed, the only information required to reconstruct the solution is the set of the time dependent coefficients $\left\{m_{i, j}(t)\right\}_{j=1}^{p_{i}}$. To that purpose, the PDE system (1)-(10) is projected over the most representative POD basis. In this work, the practical way to carry out the projection is to multiply Eqns (1)-(10) by the POD basis and integrate the result over the spatial domain. This procedure leads to the following system of ordinary differential equations:

$$
\frac{d \mathbf{m}_{i}}{d t}=\mathcal{A}_{i} \mathbf{m}_{i}+\mathbf{F}_{i},
$$

where $\mathbf{m}_{i}=\left[m_{i, 1}, m_{i, 2}, \ldots, m_{i, p_{i}}\right]^{T}$ and the subindex $i$ indicates the states described in (1)-(8). $\mathcal{A}_{i}=\int_{V} \Phi_{i}^{T}(\xi) \nabla D_{i} \nabla \Phi_{i} d \xi^{1}$ and $\mathbf{F}_{i}=\int_{V} \Phi_{i}^{T}(\xi) f_{i}(\xi) d \xi$ are, respectively, the projection of the laplacian and nonlinear terms $f_{i}$ which include both reaction and permeation terms. Finally, $\Phi_{i}=\left[\phi_{i, 1}, \phi_{i, 2}, \ldots, \phi_{i, p_{i}}\right]$ is the subset of the eigenfunctions computed from problem (17) containing the $p_{i}$ most representative basis functions. By most representative we mean those PODs basis with the largest eigenvalues $\lambda_{i, j}$. When considering real time applications, the solution of the ODE system (19) is the only task to be performed on-line.

So far, the way to compute the POD basis and its associated time dependent coefficients has been presented. Both terms are then combined, as in (16), to approximate each variable.

It should be stressed that the eigenvalues $\lambda_{i, j}$ can be employed as an $a$ priori measurement of the accuracy of the approximation (Sirovich, 1987). In this sense, the percentage of energy captured by a given number $p_{i}$ of PODs is given by:

$$
E_{i}(\%)=\frac{\sum_{j=1}^{p_{i}} \lambda_{i, j}}{\sum_{j=1}^{n} \lambda_{i, j}} 100 .
$$

\footnotetext{
${ }^{1}$ The computation of matrix $\mathcal{A}_{i}$ is also performed off-line
} 
with $n$ being the total number of eigenvalues of the discrete counterpart of Eqn (17) with the discrete kernel (18). The larger the energy captured, the better the quality of the approximation.

\subsubsection{Joint basis}

The key difference of this approach with respect to that one presented in the previous section is that a unique set of PODs containing the information for all the state variables will be computed at once. This kind of technique results particularly advantageous when highly coupling effects between system state variables are present, making the evolution of each state variable strongly connected to the evolution of the others. In the case of the pear refrigeration model such coupling comes at the large time scales from the water transport. This mechanism makes the other state variables strongly dependent on the water evolution. In this way, one unique set of time dependent coefficients encoding the evolution of all state variables should be enough to describe the system behavior.

As in the previous case, the solution of the PDE system is approximated by a truncated series expansion. However, instead of using different series for different state variables a unique series will be employed:

$$
z(\xi, t) \simeq \tilde{z}(\xi, t)=\sum_{j=1}^{p} m_{j}(t) \phi_{j}(\xi)
$$

where $\left\{\phi_{j}(\xi)\right\}_{j=1}^{p}$ is the basis set and $\left\{m_{j}(t)\right\}_{j=1}^{p}$ are the coefficients of the series decomposition.

The first step is to construct the set of snapshots employed to compute the POD basis. In this case, such set will contain the information related to all the process variables and will be constructed as a matrix $Z \epsilon \mathbf{R}^{n_{s} \cdot n_{d} \times n_{t}}$, built by collecting column-wise the vectors $Z_{i}=\left[C_{S}, C_{H}, C_{L}, C_{W}, C_{O_{2}}, C_{C O_{2}}, C_{N_{2}}, T\right]^{T}$ employed in (18). The indexes $n_{s}, n_{d}$ and $n_{t}$ represent, respectively, the number of state variables; spatial measurement points and sampling times. Remark once again that the selection of the set of snapshots will have a direct impact on the predictive capabilities of the ROM -a question that will be discussed in detail in section 3.2-.

The basis functions are computed by solving the following eigenvalue problem:

$$
\lambda_{j} \phi_{j}(\xi)=\int_{V} K\left(\xi, \xi^{\prime}\right) \phi_{j}\left(\xi^{\prime}\right) d \xi^{\prime}
$$


As in section 2.2.2, instead of working with the continuous version of the Kernel $K\left(\xi, \xi^{\prime}\right)$, we use the discrete version constructed as:

$$
K=\frac{1}{k} \sum_{n=1}^{k} Z_{n} Z_{n}^{T}
$$

where the subindex $n$ stands for a state measurement at time $t_{n}$. Finally, the original PDE system (1)-(10) is projected over the basis functions. As a result, the following ODE system is obtained:

$$
\frac{d \mathbf{m}}{d t}=\mathcal{A} \mathbf{m}+\mathbf{F}
$$

It must be noted that, when considering joint basis, only one set of time coefficients covering all the state variables is required. The original solution is recovered by combining the POD basis with the time coefficients.

\subsubsection{Nonlinear terms}

One of the difficulties in the derivation of ROMs lies in the computation of integrals of highly nonlinear terms which appear as a result of the projection procedure. In this work, the mass matrix of the FEM, $M$, will be employed to compute spatial integrals (for details see García et al. (2007)). In this sense, the projection of a given nonlinear function of state variables $f(z)$ over a given basis function can be numerically computed as:

$$
F=\Phi_{i}^{T} M \mathcal{F}
$$

where $\Phi_{i}$ and $\mathcal{F}$ are, respectively, the discrete versions of the eigenfunctions $\phi_{i}(\xi)$ and the nonlinear term $f(z)$. The product $\Phi_{i}^{T} M$ is also performed off-line.

\section{Results \& Discussion}

In this section a complete study on the numerical simulation of the pear refrigeration process on modified atmospheres using the POD technique will be performed. The geometry and the FEM spatial discretization of an average pear are presented in Figure 1. 
The FEM will be employed here to carry out three different tasks: i) to obtain the snapshots required for the derivation of the POD basis, ii) to numerically compute the spatial integrals involved in the solution of the integral eigenvalue problem and in the projection of the PDEs and iii) to check the validity of the ROM.

For the sake of clarity, it will be assumed that the pear has an homogeneous composition. However, this methodology could be also applied to heterogeneous materials. The model parameters are given in Table 2 .

The first step in the derivation of the ROM is to compute the PODs basis. To that purpose, a number of snapshots, representative of the dynamical behavior of the system, are required. As mentioned before, those snapshots were obtained by simulation using a FEM commercial software (COMSOL Multiphysics 3.5(C).

For the solution with the FEM, linear Lagrange elements were considered and a spatial mesh of 1818 points (see Figure 1(b)) was used. Further spatial refinements did not significantly improve the accuracy of the solution. The numerical integration of the resulting ODEs system was performed via a BDF (backward differentiation formula) algorithm. Note that since the model consists of eight state variables, around 14500 ODEs need to be solved with this scheme. The simulation experiments performed to obtain the snapshots included combinations of typical values of oxygen concentration, temperature and relative humidity inside the refrigeration chamber. In particular the following values were considered: $4^{\circ} \mathrm{C}, 5^{\circ} \mathrm{C}$ and $6^{\circ} \mathrm{C}$ for the chamber temperature, 95\%, $75 \%$ and $60 \%$ for the relative humidity. Finally, the air composition in the chamber is $21 \%, 2 \%, 10 \%$ for the $O_{2} .27$ simulations were ran to obtain the snapshots.

One of the main difficulties in the simulation of the refrigeration process described by equations (1)-(15) is that multiple timescales are involved (see Table 3). In this regard, some of the process variables like temperature, reach their steady state within a few hours while others like the water loss evolve in the range of months. From this perspective, one should be specially careful when choosing the snapshots to compute the POD basis. The following two sections provide a complete description of the procedure to take the snapshots for the case of separate and joint basis, respectively.

Finally, a new experiment, referred to as Exp 28, will be employed for validation of the methodologies. In this experiment the chamber temperature is $4.5^{\circ} \mathrm{C}$, while the relative humidity and the $\mathrm{O}_{2}$ concentration in the chamber's air are $80 \% \mathrm{RH}$ and $19 \% \mathrm{O}_{2}$, respectively. It should be noted that, 
although such experimental conditions are different from those considered in the computation of the snapshots, they remain within the range delimited by the 27 previous experiments.

\subsection{PODs for each variable}

In deriving the ROM, the first step consists of constructing the matrix with the state measurements (snapshots). Such measurements have been collected taking into account the different time scales of the process. The same number of snapshots, 61, have been employed for all state variables, however the sampling frequency differs from one variable to another according to their characteristic times. In this sense, snapshots for the fastest state variables ( $\mathrm{T}, \mathrm{O}_{2}, \mathrm{CO}_{2}$ and $\mathrm{N}_{2}$ ) have been taken every 5 min whereas for the remaining state variables the time interval between two consecutive measurements was around $22 \mathrm{~h}$.

Once the snapshots are available, the kernel $K$ is constructed as in Eqn (18). Since the PODs are obtained separately for each variable, different kernels $\left(K_{i}\right.$ with $\left.i=\left\{\mathrm{O}_{2}, \mathrm{CO}_{2}, T, \ldots\right\}\right)$ will result. Each of the kernels will be used in the integral eigenvalue problem whose solution will lead to a set of PODs for each state.

Given the PODs basis, the field can be reconstructed by computing the time dependent coefficients $(\mathbf{m}(t))$-see Eqn (16)-. In order to compute such coefficients, equations (1) - (10) are projected onto the corresponding POD basis set. It should be pointed out that, depending on the state dynamics, different number of PODs may be required. In this sense, those variables with stronger nonlinearity and spatial dependency like $W, \mathrm{O}_{2}$ or $\mathrm{N}_{2}$ are expected to require a larger number of PODs than those with lower spatial dependency like lamella or hexose.

The methodology described in García et al. (2007) is employed to numerically compute the spatial integrals involved in the projection procedure. Both the computation of the POD basis and the projection of the linear terms are performed off-line; therefore the time required to carry out these tasks has no influence when implementing the procedure on a real time scheme. After the projection, a set of ordinary differential equations, of the form (19), describing the evolution of the time dependent coefficients is obtained. The ODE system integration is carried out on-line. Therefore, it should be as efficient as possible to improve its performance when considering real time applications. At this moment, both the POD basis and the time dependent 
coefficients are available and can be combined, as in Eqn (16), to obtain the approximation of the field.

In order to illustrate the basics of the ROM methodology, the evolution of the first five coefficients of decomposition (16) for oxygen concentration during Exp. 28 are depicted in Figure 2. As shown in the figure, most time information is encoded in the first two coefficients, and possibly on the third one. After the third coefficient, the remaining ones converge to zero very rapidly; thus their contribution to decomposition (16) can be neglected. In dissipative systems, as the one considered in this work, only a few time-dependent coefficients $m_{O_{2}, j}$ exhibit some non-trivial dynamics while the remaining converge exponentially to zero (Vilas et al., 2007). Also the stable coefficients $m_{O_{2}, j}$ can be ordered so that the larger the value of $j$, the faster its convergence to zero and therefore the lower its contribution to the solution. This kind of behavior can be also appreciated in the coefficients associated to the rest of the state variables.

Let us now check the performance of the resulting ROM as compared with the original FEM solution by making use of Exp 28. To that purpose, a first approximation of the model (ROM1) will be developed. The number of eigenfunctions/coefficients employed to approximate the solution of the validation experiment are presented in Table 4. The maximum and mean relative errors between ROM1 and FEM solutions are, for the worst case (i.e. the $\mathrm{CO}_{2}$ ), $8.6 \%$ and $1.5 \%$ respectively. It must be pointed out that the oxygen was removed from this comparison since, under these conditions, there are several points in the pear in which the oxygen concentration are close to zero, therefore relative errors cannot be employed. For oxygen the maximum and mean absolute errors were 0.0342 and 0.0076 . ROM1 solution seems to be already in good agreement with the FEM. However, in order to improve the accuracy of the approximation, a second ROM (ROM2) was derived by increasing the number of basis functions in those variables with stronger spatial distribution (see Table 4). In this case, the maximum and mean relative errors between the FEM and ROM2 for the $\mathrm{CO}_{2}$ were $5.2 \%$ and $0.3 \%$, respectively.

Note from Table 4, that one POD is enough in both ROM1 and ROM2 for approximating starch, middle lamella and hexose concentrations. The reason for this must be found in the fact that these state variables are homogeneously distributed in space.

The evolution of temperature, oxygen and water concentration at three different spatial points is depicted in Figures 3 and 4 . Continuous lines 
represent the solution obtained with the FEM whereas marks correspond to the solution obtained with ROM2. The points represented in the figure are located in the longitudinal axis of the pear and their spatial coordinates are $p_{1}=(0,0.1073,0)$ (top of the pear), $p_{2}=(0,0.03,0)$ (around $1 / 3$ of the pear height), and $p_{3}=(0,0.07,0)$ (around $2 / 3$ of the pear height).

As shown in the figure, the ROM is in good agreement with the FEM solution. It is important to remark that the dimensionality of both ROMs is around 500 times lower than that of the FEM. The time required to perform one simulation (in an Intel $\mathrm{R}$ Core ${ }^{\mathrm{TM}}$ i7 PC using Matlab R2009 $\mathrm{R}$ ) under Linux 32-bit) using ROM2 is around 2 minutes for the large time scales and around 25 seconds for the short time scales. This should be enough for real time optimization tasks. Regarding the complete FEM model, the matrices involved are too large causing Matlab R2009® memory problems which calls

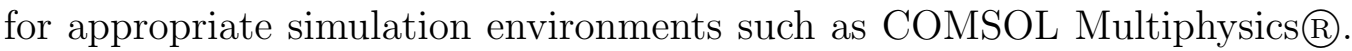
In this environment it takes around $1.5 \mathrm{~h}$ to solve the model for large time scales.

\subsection{Joint PODs}

As mentioned before, the state variables in this system are highly coupled through the reaction terms. This will allow us to derive a new ROM with a lower computational cost as compared with the separate basis ROM.

The main difference between this method and the one applied in the previous section is that a unique set of POD basis, collecting the information of all state variables, is computed. Hence, all the snapshots are arranged as explained in section 2.2.3 in order to, first, compute the kernel in Eqn. (23) and, second, solve the integral eigenvalue problem in Eqn (22). As a result a set of POD basis of dimension $\Phi \in \mathbf{R}^{n_{s} n_{d} \times n_{p}}$ is obtained. Once the POD basis are available, the procedure to compute the coefficients of the decomposition (21) is similar to the one discussed previously. More precisely system (1) - (10) is projected over the basis functions with the largest eigenvalues to obtain a set of ordinary differential equations describing the evolution of the time dependent coefficients - see Eqn (24)-.

For control purposes, large time scales are of particular interest. Thus, in a first attempt, the aim of the ROM will be to reproduce such time scale. In this context, the snapshots will be chosen along the whole process duration employing the same battery of 27 experiments as in the separated basis section. From each experiment, 24 equidistant measurements have been con- 
sidered.

Exp. 28 will be also employed in this section to validate this new ROM, using 18 PODs. A comparison between the results obtained with the FEM and this method is presented in Figures 5 (for oxygen concentration and temperature) and 6 (for water concentration). Continuous lines correspond with FEM while marks represent the ROM solution. As in the previous case, the points chosen for the representation are $p_{1}=(0,0.1073,0), p_{2}=$ $(0,0.03,0)$, and $p_{3}=(0,0.07,0)$.

The figures show that, when considering large time scales (several days), the ROM results are in good agreement with the FEM.

Note that, when perturbations enter the process (for instance when the door of the refrigeration chamber is open), short times scales should be considered. However since no information concerning such time scales was included in the snapshot set, the ROM will not be able to reproduce the real behavior. For example, the temperature, under the absence of further perturbations, becomes spatially homogeneous within 5 to 7 hours and the first snapshot is taken after $15 \mathrm{~h}$. The resulting set of measurements, to be employed in the computation of the POD basis, will thus not contain any information about its spatial distribution. As shown in Figure 7(a), the ROM will provide a solution which is spatially homogeneous, i.e., the state variables have the same value at all spatial points.

Short time scales may be reproduced by increasing the sampling frequency. In this regard, when the basis functions are computed from a set of 61 snapshots per experiment, collected every 5 minutes, the FEM behavior is recovered by using only 18 PODs in the projection (see Figure 7b). However, the ROM will find difficulties when trying to reproduce larger time scales due to the absence of data for such scales.

From previous discussion, it seems reasonable to combine measurements taken at all time scales. To that purpose, the characteristic times of the most relevant phenomena occurring in the system (respiration, gas, water and heat transport, starch hydrolysis,...) were identified and different sampling frequencies were chosen accordingly. In this case, all the time scales can be recovered with the ROM as shown in Fig 8. However, the price to pay is that a larger number of PODs (in this case 34) must be employed in the projection. Note that 34 basis functions is within the order of the separate basis ROM.

In this case, the time required to perform one simulation is around 1 minute for the large time scales and around 10 seconds for the short time 
scales (half of the time employed in the separate basis case). Note also that the versions of the codes were not optimized and the efficiency could be largely improved by carefully selecting the IVP integration methods and the simulation environment (for instance using FORTRAN).

The results obtained so far suggest that an adaptive model which changes its dimensionality according to a given criteria could provide an intermediate solution between joint and separate basis. The main idea would be that, at the beginning of the process or when significant perturbations occur in the system, the ROM with a larger number of PODs should be employed while when the effects of such perturbations have passed, one should switch to the ROM with a lower number of PODS.

\section{Conclusions}

The work focused on the derivation of efficient yet accurate mathematical models to be employed in a real time monitoring/control scheme. The aim of such scheme is to preserve the fruit quality during refrigeration and transportation.

First, we make use of detailed nonlinear and spatially distributed mathematical models developed in the literature and defined on an irregular 3D spatial domain. Such models describe the different phenomena involved in the refrigeration process and have been validated through experimental data. We showed in this work that classical numerical methods like the FEM are computationally too involved (it takes around $1.5 \mathrm{~h}$ to perform one model simulation) and therefore they are unsuitable for real time applications because of the high dimensionality of the resulting ODE system.

As an alternative to the FEM, we proposed the use of reduced order models, and in particular the POD technique due to its proven efficiency. Two different approaches have been considered. The first, defined as separate basis, leads to one ROM for each of the state variables. The resulting model consisted of 28 ODEs (a size more than 500 times lower than in the FEM case). The second approach (joint basis) takes advantage of the coupling effects between the different state variables to reduce even more the dimensionality of the ROM. The difference with respect to the first one is that the evolution of all the state variables is described by the same system of ODEs.

The joint basis technique has proven to be more efficient when dealing with problems with only one significant time scale. However, when the pro- 
cess variables evolve at different time scales and all of them must be considered, the efficiency of this technique is comparable to the separate basis.

These results suggest the construction of an adaptive model which automatically would select the appropriate model dimensionality according to the time scale should be considered.

\section{References}

Alonso, A. A., García, M. R., And Vilas, C. 2010. Dynamic Modeling and Simulation for Robust Control of Distributed Processes and Bioprocesses. In Process System Engineering; Dynamic Process Modelling, 7, (E.N. Pistikopoulus, M.C. Georgiadis, V. Dua, eds.) pp 369-397, WileyVCH, Germany.

Balsa-Canto, E., Alonso, A. A., and Banga, J. R. 2002. A novel, efficient and reliable method for thermal process design and optimization. Part II: Applications. J. Food Eng., 52, 235-247.

Datta, A. K. 2007. Porous media approaches to studying simultaneous heat and mass transfer in food processes I: Problem formulations. J. Food Eng., 80, 80-95.

De Smedt, V., Barreiro, P., Verlinden, B. E., Veraverbeke, E. A., De Baerdamaeker, J., And Nicolaï, B. M. 2002. A mathematical model for the development of mealiness in apples. Postharv. Biol. Technol., 25, 273-291.

Fletcher, C. A. J. 1984. Computational Galerkin Methods. SpringerVerlag, New York.

García, M. R., Vilas, C., Banga, J. R., and Alonso, A. A. 2007. Optimal field reconstruction of distributed process systems from partial measurements. Ind. Eng. Chem. Res., 46, 530-539.

García, M. R., Vilas, C., Santos, L. O., And Alonso, A. A. 2012. A robust multi-model predictive controller for distributed parameter systems. J. Process Control, 22, 60-71. 
Gottlieb, D. And Orszag, S. A. 1977. Numerical Analysis of Spectral Methods: Theory and Applications. Society for Industrial and Applied Mathematics, Philadelphia, Pennsylvania.

Gunes, H. 2002. Low-order dynamical models of thermal convection in high-aspect ratio enclosures. Fluid Dyn. Res., 30, 1-30.

Holmes, P. J., Lumley, J. L., Berkooz, G., Mattingly, J. C., And WittenberG, R. W. 1997. Low-dimensional models of coherent structures in turbulence. Phys. Rep., 287, 338-384.

Lammertyn, N., Scheerlinck, N., Jancsó, P., Verlinden, B. E., AND NicolaÏ, B. M. 2003. A respiration-diffusion model for conference pears I: model development and validation. Postharv. Biol. Technol., 30, 29-42.

Martínez-Monzo, J., Barat, J. M., GonzÁlez-Martínez, C., Ciralt, A., And Fito, P. 1999. Changes in thermal properties of apple due to vacuum impregnation. J. Food Eng., 43, 213-218.

Nguyen, T. A., Dresselaers, T., Verboven, P., Dhallewin, G. D., Culeddu, N., Hecke, P. V., And Nicolaï, B. M. 2006. Finite element modelling and MRI validation of 3D transient water profiles in pears during postharvest storage. Food Agric., 86, 745-756.

Padhi, R. And Balakrishnan, S. 2003. Proper orthogonal decomposition based optimal neurocontrol synthesis of a chemical reactor process using approximate dynamic programing. Neural Networks, 16, 719-728.

Park, H. M. And Chung, O. Y. 2000. Reduction of modes for the solution of inverse natural convection problems. Comput. Methods Appl. Mech. Engrg., 190, 919-940.

Reddy, J. N. 1993. An Introduction to the Finite Element Method. McGraw-Hill, New York. 2nd edition.

Rico-Martínez, R., Krischer, K., And Kevrekidis, Y. G. 1995. Nonlinear system identification using neural networks: dynamics and instabilities. In Neural Networks in Chemistry and Chemical Engineering. (A. Bulsari). Elsevier. 
Schiesser, W. E. 1991. The Numerical Method of Lines. Academic Press, New York.

Sirovich, L. 1987. Turbulence and the dynamics of coherent structures. Part I: Coherent structures. Quaterly of Appl. Math., 45, 561-571.

Theodoropoulou, A., Adomaitis, R. A., And Zafiriou, E. 1998. Model reduction for optimization of rapid thermal chemical vapor deposition systems. IEEE Trans. on Semiconduct. Manufact., 11, 85-98.

Tri Ho, Q., Verboven, P., Verlinden, B. E., Lammertyn, J., Vandewalle, S., And Nicolä̈, B. M. 2008. A continuum model for metabolic gas exchange in pear fruit. PLOS Comput. Biol., 4, 1-13.

Tri Ho, Q., Verlinden, B. E., Verboven, P., Vandewalle, S., And Nicolä̈, B. M. 2006. A permeation-diffusion-reaction model of gas transport in cellular tissue of plant materials. J. Exp. Bot., 57, 4215-4224.

Vilas, C., García, M. R., Banga, J. R., and Alonso, A. A. 2006. Stabilization of inhomogeneous patterns in a diffusion-reaction system under structural and parametric uncertainties. J. Theor. Biol., 241, 295-306.

Vilas, C., García, M. R., Banga, J. R., and Alonso, A. A. 2007. Robust feed-back control of distributed chemical reaction systems. Chem. Eng. Sci., 62, 2941-2957.

Vilas, C., García, M. R., Banga, J. R., and Alonso, A. A. 2008. Robust feed-back control of travelling waves in a class of reaction-diffusion distributed biological systems. Physica D: Nonlinear phenomena, 237, 2353-2364. 


\section{Figures}

(a)

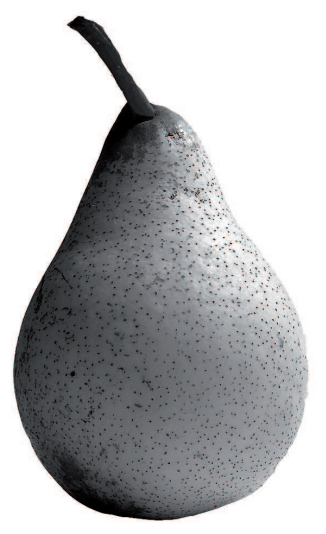

(b)

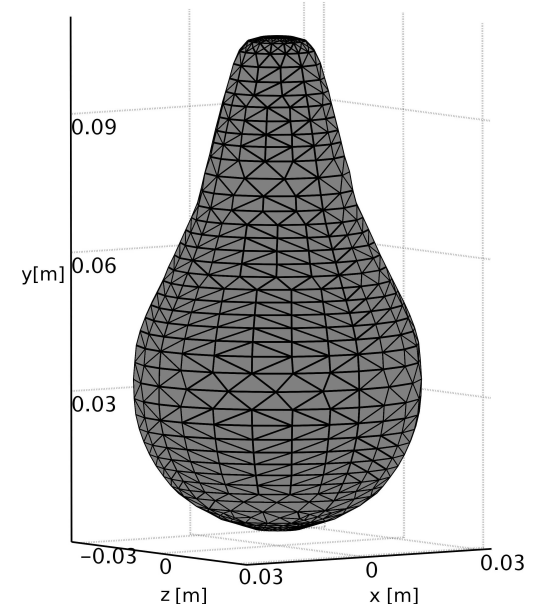

Figure 1: (a) Real pear. (b) Geometry and FEM spatial discretization of the pear considered in this work. The geometry of the pear has been obtained from Tri Ho et al. (2008).

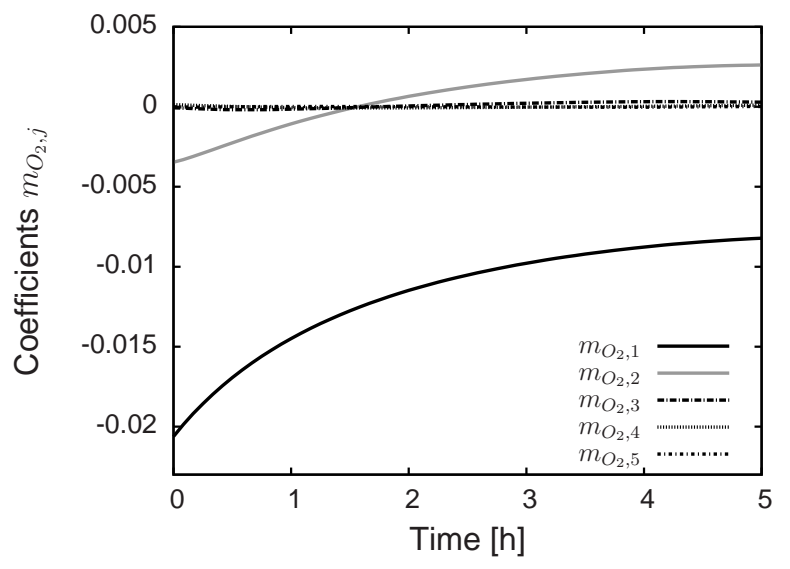

Figure 2: Time dependent coefficients for oxygen concentration, $m_{\mathrm{O}_{2}, j}(t)$. 
(a)

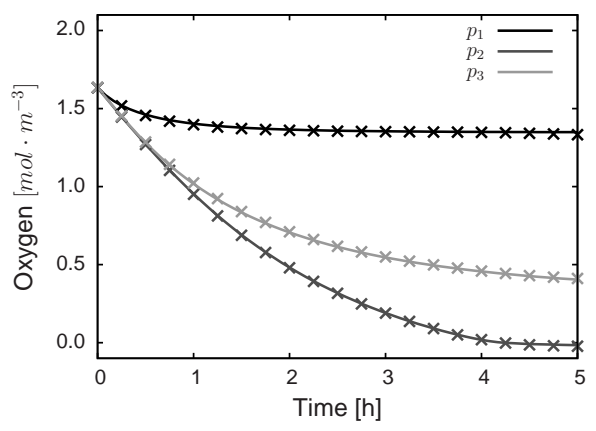

(b)

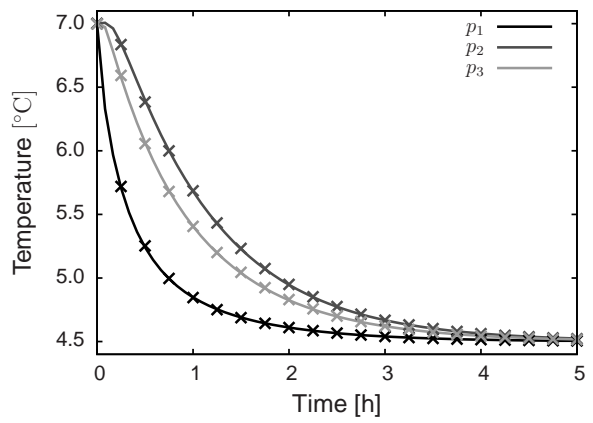

Figure 3: Comparison between FEM (continuous lines) and ROM2 (marks) solutions for Exp. 28. (a) $\mathrm{O}_{2}$ concentration and (b) temperature.

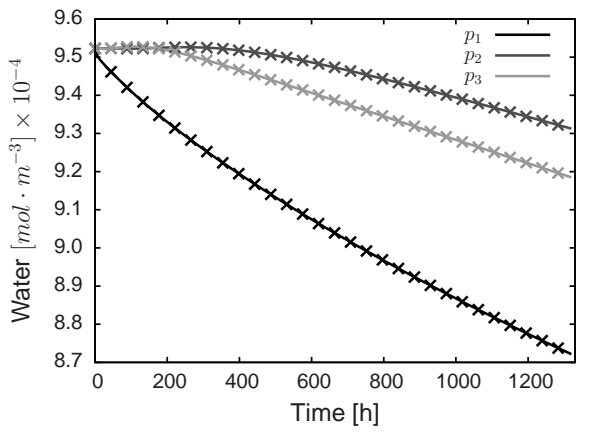

Figure 4: Comparison between FEM (continuous lines) and ROM2 (marks) solutions for Exp. 28 for water concentration. 
(a)

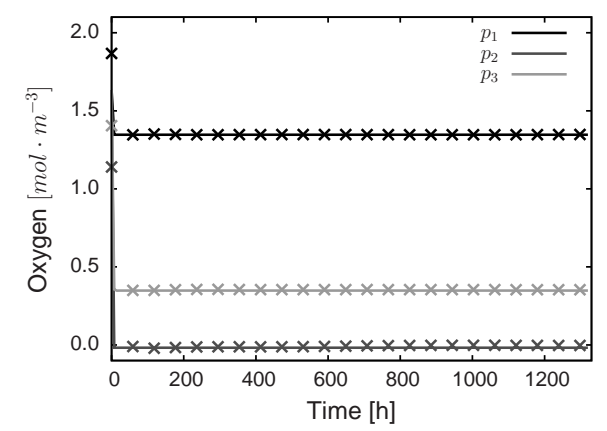

(b)

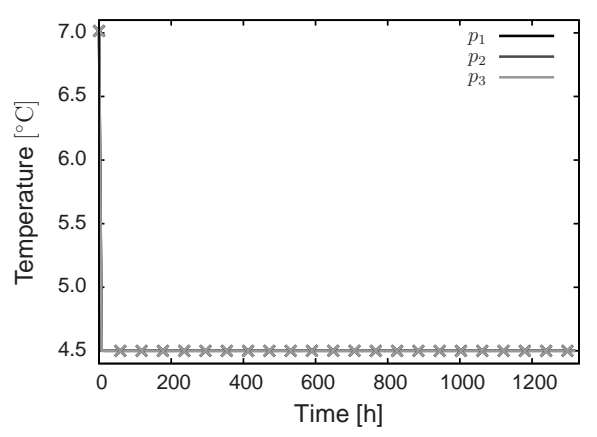

Figure 5: Comparison between FEM (continuous lines) and ROM (marks) solutions for (a) $\mathrm{O}_{2}$ concentration and (b) temperature.

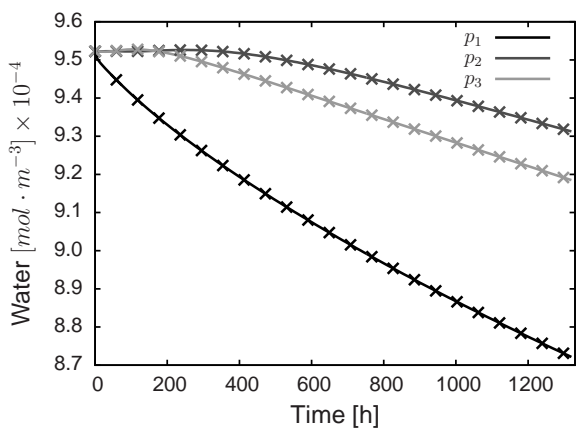

Figure 6: Comparison between FEM (continuous lines) and ROM (marks) solutions for water concentration. 
(a)

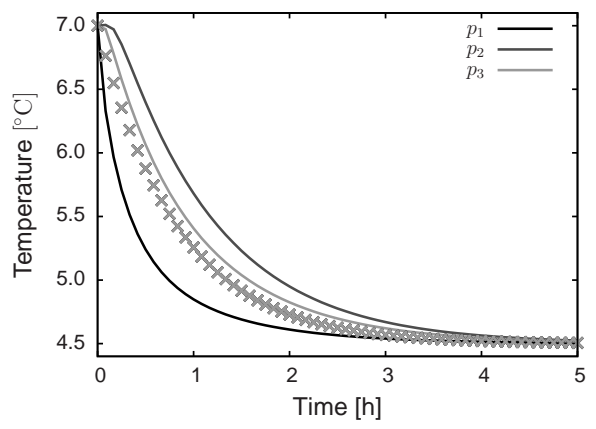

(b)

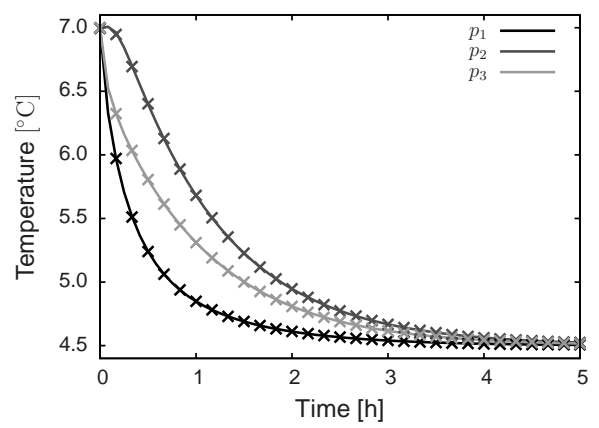

Figure 7: Comparison between FEM (continuous lines) and ROM (marks) solutions for $T$ at short time scales using measurements of (a) large time scales and (b) short time scales.

(a)

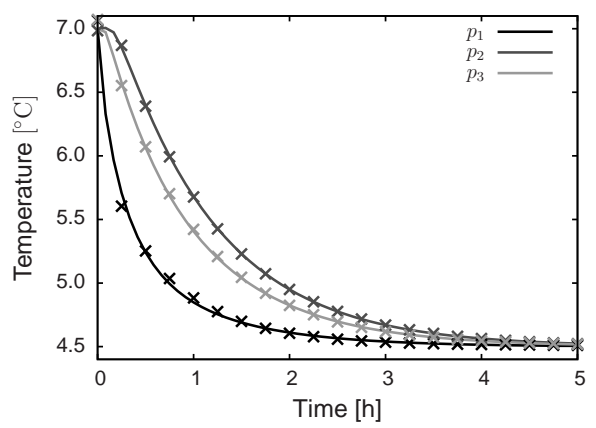

(b)

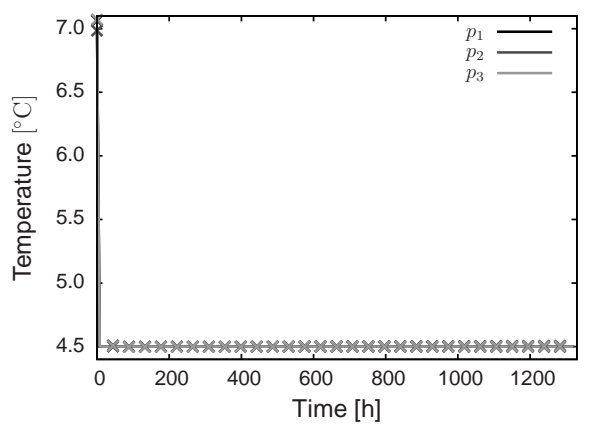

Figure 8: Comparison between FEM (continuous lines) and ROM (marks) solutions for $\mathrm{T}$ combining measurements of different times scales. Solutions at (a) short time scales and (b) large time scales. 


\section{Tables}

\begin{tabular}{|c|c|c|}
\hline Constant & Units & Description \\
\hline$\overline{k_{S}}$ & $s^{-1}$ & Rate constant for starch \\
\hline$k_{L}$ & $s^{-1}$ & Rate constant for middle lamella \\
\hline$n_{S}$ & - & $\begin{array}{l}\text { Number of molecules of hexose in a molecule of } \\
\text { starch }(\sim 530)\end{array}$ \\
\hline$E_{a, V m, O_{2}}$ & $\mathrm{Jmol}^{-1}$ & Activation energy for $\mathrm{O}_{2}$ consumption \\
\hline$E_{a, V m f C O_{2}}$ & $\mathrm{Jmol}^{-1}$ & $\begin{array}{l}\text { Activation energy for fermentative } \mathrm{CO}_{2} \text { produc- } \\
\text { tion }\end{array}$ \\
\hline$R$ & Jmol $^{-1} K^{-1}$ & Universal gas constant \\
\hline$V_{m, O_{2}, r e f}$ & $m o l m^{-3} s^{-1}$ & $\mathrm{O}_{2}$ consumption rate \\
\hline$R Q$ & - & Cellular respiration quotient $(R Q=1)$ \\
\hline$V_{C O_{2, r e f}}$ & $m o l m^{-3} s^{-1}$ & $\mathrm{CO}_{2}$ consumption rate \\
\hline$K_{m, O_{2}}$ & molm $^{-3}$ & Michaelis-Menten constant for $\mathrm{O}_{2}$ consumption \\
\hline$K_{m n, C O_{2}}$ & molm ${ }^{-3}$ & $\begin{array}{l}\text { Michaelis-Menten constant for non competitive } \\
\mathrm{CO}_{2} \text { inhibition }\end{array}$ \\
\hline$K_{m, f, O_{2}}$ & molm ${ }^{-3}$ & $\begin{array}{l}\text { Michaelis-Menten constant of } \mathrm{O}_{2} \text { inhibition on } \\
\text { fermentative } \mathrm{CO}_{2} \text { production }\end{array}$ \\
\hline
\end{tabular}

Table 1: Constants involved in the chemical reaction terms. 


\begin{tabular}{ccc|ccc}
\hline Parameters & Value & Units & Parameters & Value & Units \\
\hline$D_{O_{2}}$ & $5 \times 10^{-9}$ & $\mathrm{~m}^{2} \mathrm{~s}^{-1}$ & $k_{S}$ & $7.5 \times 10^{-7}$ & $\mathrm{~s}^{-1}$ \\
$D_{C O_{2}}$ & $4 \times 10^{-8}$ & $\mathrm{~m}^{2} \mathrm{~s}^{-1}$ & $k_{L}$ & $7 \times 10^{-8}$ & $\mathrm{~s}^{-1}$ \\
$D_{N_{2}}$ & $2.67 \times 10^{-8}$ & $\mathrm{~m}^{2} \mathrm{~s}^{-1}$ & $V_{m, O_{2}, \text { ref }}$ & $2.39 \times 10^{-4}$ & $\mathrm{molm}^{-3} \mathrm{~s}^{-1}$ \\
$D_{W}$ & $6 \times 10^{-7}$ & $\mathrm{~m}^{2} \mathrm{~s}^{-1}$ & $V_{C O_{2, r e f}}$ & $1.61 \times 10^{-4}$ & $\mathrm{molm}^{-3} \mathrm{~s}^{-1}$ \\
$K_{T}$ & 0.38 & $\mathrm{WK}^{-1} \mathrm{~m}^{-1}$ & $E_{a, V m, O_{2}}$ & $8.02 \times 10^{4}$ & $\mathrm{Jmol}^{-1}$ \\
$c_{T}$ & $3.8 \times 10^{3}$ & $\mathrm{JK}^{-1} \mathrm{~kg}^{-1}$ & $E_{a, V m f, C O_{2}}$ & $5.67 \times 10^{4}$ & $\mathrm{~s}^{-1}$ \\
$\rho$ & 769.6 & $\mathrm{kgm}^{-3}$ & $K_{m, O_{2}}$ & $3 \times 10^{-3}$ & $\mathrm{molm}^{-3}$ \\
$h_{O_{2}}$ & $6 \times 10^{-7}$ & $m s^{-1}$ & $K_{m n, C O_{2}}$ & 14.51 & $\mathrm{molm}^{-3}$ \\
$h_{C O_{2}}$ & $7.5 \times 10^{-7}$ & $m s^{-1}$ & $K_{m, f, O_{2}}$ & $2.83 \times 10^{-4}$ & $\mathrm{molm}^{-3}$ \\
$h_{N_{2}}$ & $6 \times 10^{-7}$ & $m s^{-1}$ & $R$ & 8.314 & $\mathrm{Jmol}^{-1} \mathrm{~K}^{-1}$ \\
$h_{W}$ & $1.59 \times 10^{-10}$ & $\mathrm{~ms}^{-1}$ & $R Q$ & 1 & - \\
$h_{T}$ & 10 & $W K^{-1} \mathrm{~m}^{-2}$ & $k_{p}$ & $2.35 \times 10^{-19}$ & $\mathrm{~m}^{2}$ \\
$\alpha_{C_{O_{2}}}$ & 0.2532 & - & $\alpha_{C_{C O}}$ & 0.8254 & - \\
$\alpha_{C_{N_{2}}}$ & 0.2411 & - & & & \\
\hline
\end{tabular}

Table 2: Parameters of the model.

\begin{tabular}{lccccc}
\hline & Diffusion & Starch react. & Lamella react. & Cellular resp. & Fermentation \\
\hline$C_{W}$ & $3-4$ months & $2-3$ weeks & $4-6$ months & $2-5$ hours & - \\
$C_{\mathrm{O}_{2}}$ & $15-20$ hours & - & - & $2-5$ hours & - \\
$C_{\mathrm{CO}_{2}}$ & $10-15$ hours & - & - & $10-15$ hours & $3-5$ months \\
$C_{\mathrm{N}_{2}}$ & $15-20$ hours & - & - & - & - \\
$T$ & $2-5$ hours & - & - & - & - \\
\hline
\end{tabular}

Table 3: Time required, considering reference conditions, to obtain a substantial variation in the corresponding state variable as an effect of a given phenomena (characteristic times).

\begin{tabular}{lccccccccc}
\hline & & $C_{S}$ & $C_{H}$ & $C_{L}$ & $C_{W}$ & $C_{O_{2}}$ & $C_{C O_{2}}$ & $C_{N_{2}}$ & $T$ \\
\hline Number of & ROM1 & 1 & 1 & 1 & 6 & 3 & 2 & 6 & 2 \\
POD modes & ROM2 & 1 & 1 & 1 & 8 & 6 & 3 & 8 & 3 \\
\hline
\end{tabular}

Table 4: Number of POD modes employed in the different ROMs and in each state variable. 


\section{Acknowledgments}

This work has been partially financed by the Spanish Ministry of Science and Innovation projects (AGL2008-05267-C03-01 and MTM2010-21135-C02-02), Xunta de Galicia project IN845B-2010/046 and FP7 CAFE project (KBBE2007-1-212754). The authors would also like to acknowledge prof. Bart Nicolaï and Dr. Quang Tri Ho for providing us with the pear geometry. 